\title{
Análisis de la vegetación del páramo del Duende, Chocó
}

\section{Analysis of the vegetation of the páramo del Duende, Choco}

\author{
Giovanny Ramírez Moreno, Bıol, MSc ${ }^{1}$, Zulmary Valoyes Cardozo, Bıol ${ }^{2}$
}

\section{RESUMEN}

La flora establecida en el páramo del Duende corresponde a especies vegetales que revisten suma importancia para el establecimiento y colonización de una serie de diferentes formas de vida dentro de este ecosistema. Con el objetivo de analizar la vegetación presente en el único páramo hasta ahora reportado para el departamento del Chocó, se levantó primero una línea base de información existente y en campo se hicieron observaciones directas en tres unidades paisajísticas localizadas en el humedal Citará y los nacimientos de los ríos Copoma y Cucurrupí. El muestreo se realizó al azar y se colectaron individuos de preferencia de plantas fértiles para su posterior identificación. Para definir la estructura de la población de Espeletia frontinoensis se delimitó un transecto de $25 \times 4 \mathrm{~m}$, equivalente a $100 \mathrm{~m}^{2}$, en donde se contaron todos los individuos correspondientes a esta población, en diferentes estados sucesionales (plántula, juvenil y adulto). Se logró documentar la composición, estructura y análisis de la vegetación de este páramo, y se dio a conocer un grupo significativo de plantas nunca antes publicadas para la región, dando un aporte para el conocimiento, uso y conservación del ecosistema.

Palabras clave: Páramo; Composición; Estructura.

\section{ABSTRACT}

The flora established in the páramo del Duende corresponds to vegetal species that have with extreme importance for the establishment and colonization of a series of differentforms from life within this ecosystem. Because analyzing the present vegetation in the unique moorland now reported for the Department of Choco, I rise initially to a line base of existing information and in field direct observations in three located landscaping units in Citara moist soil and the rivers Copoma and Cucurrupi. The sampling I am realised at random and individuals of preferably fertile seedlings for their later identification collected themselves. In order to define the structure of the population of Espeletia frontinoensis plot of $25 \times 4 \mathrm{~m}$ was delimited, equivalent to $100 \mathrm{~m}^{2}$, where all the individuals corresponding to this one counted themselves, in different sucecionales states (seedling, juvenile and adult). It was managed to document the composition, it structures and analysis of the vegetation of this moorland, and one occurs to know a significant group plants never before published for the region, giving a contribution for the knowledge, use and conservation of the ecosystem.

Keywords: Moorland; Composition; Structure.
1. Investigador principal Componente Ecosistémico, Instituto de Investigaciones Ambientales del Pacífico, Quibdó, Colombia.

e-mail:gramirezm3@hotmail.com

2. Docente, Universidad Tecnológica del Chocó, Quibdó, Colombia. email: zulmaryvaloyes@hotmail.es Grupo de investigación en Conocimiento, manejo y conservación de los ecosistemas delChocó biogeográfico.

Recibido: Marzo 25, 2010 Aceptado: Abril 15, 2010 


\section{INTRODUCCIÓN}

Hasta la fecha existe un gran vacío de información acerca de la estructura y composición florística del ecosistema paramuno presente en el departamento del Chocó, porque han sido escasos o casi nulos los estudios realizados en esta zona. Teniendo en cuenta trabajos como el inventario realizado para la elaboración del plan de manejo del páramo del Duende en la jurisdicción correspondiente al Valle del Cauca, se ha podido estimar que está franja se encuentra entre 34003800; en el Duende se han identificado 275 especies de plantas vasculares, 145 géneros y 71 familias. Las familias predominantes son Asteraceae, Orchidaceae y Ericaceae, seguidas de Melastomataceae y Poaceae(Vargas \& Gómez 2005). Entre las especies más representativas de este ecosistema se registran pastos (Calamagrostis, Cortadeira, Fetusca), cortaderas (Rhynchospora), hierbas de hojas arrosetadas (Plantago, Werneria, Oritrophium, Valeriana, Paepalanthus), hierbas erectas de las familias Gentianaceae y Scrofulariaceae, y hierbas pequeñas asociadas con el pastizal (Nertera, Myrteola, Viola, Disterigma, Oreobolus, Xyris). Se encuentran también algunos arbustos dispersos (Asteraceae, Rosaceae, Ericaceae, y Pericaceae y Melastomataceae). La especie arbustiva más representativa es Espeletia frontinoensis (frailejón), Blechnum sp. (helecho), Epidendrum sp. (Orchidaceae), Neurolepis sp. (pastos) Guzmania sp. (Bromeliaceae), Anturio sp. (Araceae) y familias como: Asteraceae, Ericaceae, Loranthaceae, anturios, musgos, hepáticas y líquenes. Entre las especies arbóreas más comunes se encuentran pinos colombianos, laureles de cera Myrica sp., mano de oso (Oreopanax sp., tibar sp. (Escallonia), Miconia sp. (nigüitos), Weinmannia sp. (encenillos), Tibouchina sp. (sietecueros) (CVC-FEDENA 2000).

Por otro lado Rangel-Ch. (2000) registra para el páramo del Duende Bomarea linitifolia, B. floribunda (Alstroemeriaceae), Grecia nibegena, Guzmania confinis, Racinaea tetranthta, Tillansia compacta, Maytenus novagratensis, Sisyrinchum trinerve, Epidendrum fimbriatum, Panchyphyllum verrucosum, Pleurothallis cassidis, Loricaria complanata, Monticalia arbutifolia, M. vacaccionoides, M. andicola. De las 275 especies registradas para el páramo del Duende, 26 se encuentran bajo algún grado de amenaza, 11 especies se encuentran en status S1 (especie muy amenazada): Aniba perutilis, Aphelandra acanthus, Bomarea racemosa, Chusquea latifolia, Mandevilla subsaggittata, Nectandra acutifolia (laurel amarillo), Psamisia aff., Macrophylla, Nectandra sp. (bongo amarillo), Guatteria aff. crassipes, Magnolia hernandezii, Peperomia obtusifolia; 11 especies en S2 (especie amenazada): Ladenbergia magniflora (azuceno), Cavendishia adenophora (quereme), Columnea diminata, Hedyosmum bonplandianum (silvio-silvio), Hydragnea aff. Oerostedii, Renealmia aff. cuatrecasana,
Shefflera morotoni (mano de oso), Guatteria goudotiana*, Guateria aff. crasipes, Quercus humboldtii* (roble), Weinmannia pubescens (encenillo); una especie en S3 (especie rara o con frecuencia amenazada): Guatteria aff. crassipes; dos especies en EN (en peligro): Brosimun utile (sande), Magnolia hernandezii (copachí o molinillo); una especie en NT (casi amenazado): Spirotheca rodhstyla (palo santo); una en VU (vulnerable): Spirotheca rodhostyla; tres especies en apéndice II de cites: Dichaea sp., Cyathea caracasana (palma sarro o palma baba), Pleurothallis sp. (CVC-FEDENA 2000).

Con este trabajo se logró analizar la vegetación del páramo del Duende como una estrategia para planificar el manejo y la conservación del ecosistema paramuno más conservado y desconocido del planeta.

\section{ÁREADE ESTUDIO}

El páramo del Duende se encuentra ubicado en la cordillera Occidental en los departamentos de Chocó, municipio del litoral del San Juan con un área correspondiente a $82 \%$ y Valle del Cauca (municipios de Riofrío, Trujillo y Calima-Darién) con unárea correspondiente a 18\% (CVC-FEDENA 2000); en la actualidad se considera como el páramo más intrigante del país, porque su ubicación geográfica lo convierte en un sitio casi impenetrable, situación favorable para los organismos residentes del ecosistema; la red hídrica es de vital importancia para la población asentada en los municipios cercanos. La intervención antrópica en este ecosistema es muy poca, por lo que sus bosques se conservan naturales en jurisdicción del Chocó.

En el complejo del Duende se origina la cuenca del río San Juan y Munguidó, y la cuenca del río Calima, que confluye hacia el río San Juan; por esta razón se considera importante en la regulación de aguas del Chocó biogeográfico (IDEAM 2006). Teniendo en cuenta que los valores de precipitación descienden de manera gradual a lo largo del gradiente altitudinal y que éste a su vez determina diferentes condiciones ambientales del ecosistema paramuno y hasta en la cultura de asentamientos humanos, se puede aducir que la temperatura del complejo del Duende oscila entre $\operatorname{los} 5^{\circ} \mathrm{Cy} 13^{\circ} \mathrm{C}$. La humedad relativa con frecuencia es muy alta, entre $80 \%$ y $98 \%$ durante gran parte de la noche y el día; posee un carácter variable y estacional (máxima en época de lluvias y mínima en las estaciones secas) y además se suele presentar el fenómeno de niebla.

En términos generales, el complejo se caracteriza por un paisaje muy montañoso, de laderas disectadas y pendientes muy pronunciadas donde en la medida en que aumenta la altitud se presentan áreas de intensa neblina y una superficie con un relieve ondulado de pendientes suaves y pequeños valles (Fedena et al. 2004). En la zona media de las cabeceras 


\section{Bioetnia Volumen 7 No 2 (julio-diciembre), 2010}

de los ríos Cucurrupí, Copomá y Munguidó, se encuentran temperaturas de $11^{\circ} \mathrm{C}-15^{\circ} \mathrm{C}$, frío húmedo-superhúmedo, mientras que en las zonas altas donde nacen estos ríos, las condiciones climatológicas son adversas en relación con el resto del municipio, pues se encuentran temperaturas de $7^{\circ} \mathrm{C}$ $15^{\circ} \mathrm{C}$ (frío-muy frío).

\section{METODOLOGÍA}

Fase de campo. Los sitios de muestreos se seleccionaron a través de observaciones directas, con la ayuda de la cartografía existente, se identificaron tres unidades paisajísticas: humedal Citará, ubicado dentro de las coordenadas: $4^{\circ} 04^{\prime} 0.39^{\prime}$ ', $\mathrm{N}$ y $76^{\circ} 030^{\prime} 30.29^{\prime}$ ' O , a $3.561 \mathrm{msnm}$ aproximadamente; río Cucurrupí (nacimiento) ubicado dentro de las coordenadas: 404'19.6" $\mathrm{Ny} 76^{\circ} 030$ '30.5' O a una altura aproximada de 3514 msnm y río Copomá (parte alta), dentro de las coordenadas $4^{\circ} 04^{\prime} 25^{\prime}$ ' $\mathrm{Ny} 76^{\circ} 030^{\prime} 47^{\prime}$ ' $\mathrm{O}$, a una altura aproximada de 3424 msnm.

El muestreo se realizó al azar dentro y fuera deáreas donde se pudieran integrar los componentes (agua, vegetación y fauna), tratando de recopilar la mayor información posible sobre vegetación. Se colectaron individuos de plantas presentes en cada una de las unidades muestrales, de los cuales se seleccionó con preferencia, material fértil para su posterior identificación, y se observó y anotó el hábito de crecimiento de cada una de las especies presentes. El material colectado se prensó, se etiquetó, se secó y por último se depositó en el herbario (CHOCO) de la Universidad Tecnológica del Chocó «Diego Luis Córdoba», Quibdó.

Para definir la estructura de la población Espeletia frontinoensis se delimitó un transepto de $25 \times 4 \mathrm{~m}$, equivalente a $100 \mathrm{~m}^{2}$, en donde se contaron todos los individuos correspondientes a esta población, en diferentes estados sucesionales (plántula, juvenil y adulto).

Fase de laboratorio. El material colectado se trasladó al laboratorio de Botánica y Ecología de la UTCH, donde una vez descrito y secado, se confrontó con claves taxonómicas especializadas y se comparó con algunos herbarios virtuales como el New York Botanicals Garden (NY), Neotropical Herbarium Specimens http://fm.fieldmuseum.org/vrrc, entre otros sitios disponibles; cada una de las especies se describió mediante la metodología clásica que se usa en taxonomía.

\section{RESULTADOSYDISCUSIÓN}

Composición florística. Los resultados del estudio florístico muestran la presencia de 100 especies distribuidas en 81 géneros y 43 familias. Las angiospermas estuvieron representadas por 78 especies, agrupadas en 69 géneros y 35 familias, y los pteridofitos representados por 12 familias, 16 géneros y 22 especies.
Las familias mejor representadas corresponden a: Asteraceae con 12 especies, Ericaceae y Orchidaceae con siete especies, Poaceae y Ciperaceae con cuatro especies, seguida de Rosaceae, Scrofulareaceae, Bromeliaceae y Rubiaceae con tres especies cada una, que están distribuidas en los diferentes sitios de muestreos de este ecosistema (Gráfica 1).

Flora asociada con el ecosistema. Las características ecosistémicas del ambiente paramuno muestran una representación florística demarcada por el dominio de especies representativas de estos ecosistemas diferenciadas por varios tipos de cobertura y asociaciones vegetales:

Vegetación abierta. Representada por elementos típicos de páramos donde predominan especies como Espeletia frontinoensis (frailejón) y Paepalanthus colombianun, y pastos de las familias Poaceae y Cyperaceae, con un estrato restante representado por helechos de porte herbáceo, musgo y hepáticas (Figura 1).

La estructura poblacional de frailejones en un levantamiento de $100 \mathrm{~m}^{2}$ fue de 581 individuos entre plántulas con 275 individuos, juveniles con 237 individuos y adultos 69 individuos, con lo que se evidencia la estabilidad natural de la población y su representatividad en el ambiente; además, se demarca una amplia representatividad del estado de desarrollo (plántula) de la población, indicando el proceso de colonización y dominio futuro de la especie en el ecosistema (Gráfica 2).

Vegetación arbustiva. Representada sobre todo por arbustos achaparrados donde predominan especies de Baccharis macrantha, Diplostephium sp., Hypericum sp., Coniza bonariensis, helechos arbustivos Blechum sp., entremezclados con algunos individuos de Espeletia frontinoensis y pastos de las familias Poáceas y Ciperáceas (Figura 2).

Las asociaciones predominantes en el páramo del Duende corresponden a:

Asociación Espeletio frontinoensis. Compuesta por frailejón mezclado con pastos. En su estructura se diferencian dos estratos, un estrato herbáceo donde predominan los pastos Camalagrostis efussa, Cotadeira sp., Fetusca sp., Rhynchospora sp., hierbas erectas como Halenia drasyantha, Castilleja fisifolia; hierbas arrosetadas como Valeriana bracteata, Plantago rïgida, Paepalanthus colombianun; hierbas de pequeño tamaño como Myrteola sp. y Disterigma empetrifolium, entre otras. En el estrato arbustivo predominan Loricaria complanata, Baccharis macrantha, Pernettya próstata. Se establecen en pendientes suaves y terrenos planos cercanos a las fuentes hídricas, en donde los vientos son menores, los suelos presentan poco drenaje y la humedad es mayor.

Asociación Guzmania sp., Epidendrum frutex. Esta asociación se establece en pendientes en donde el viento es 


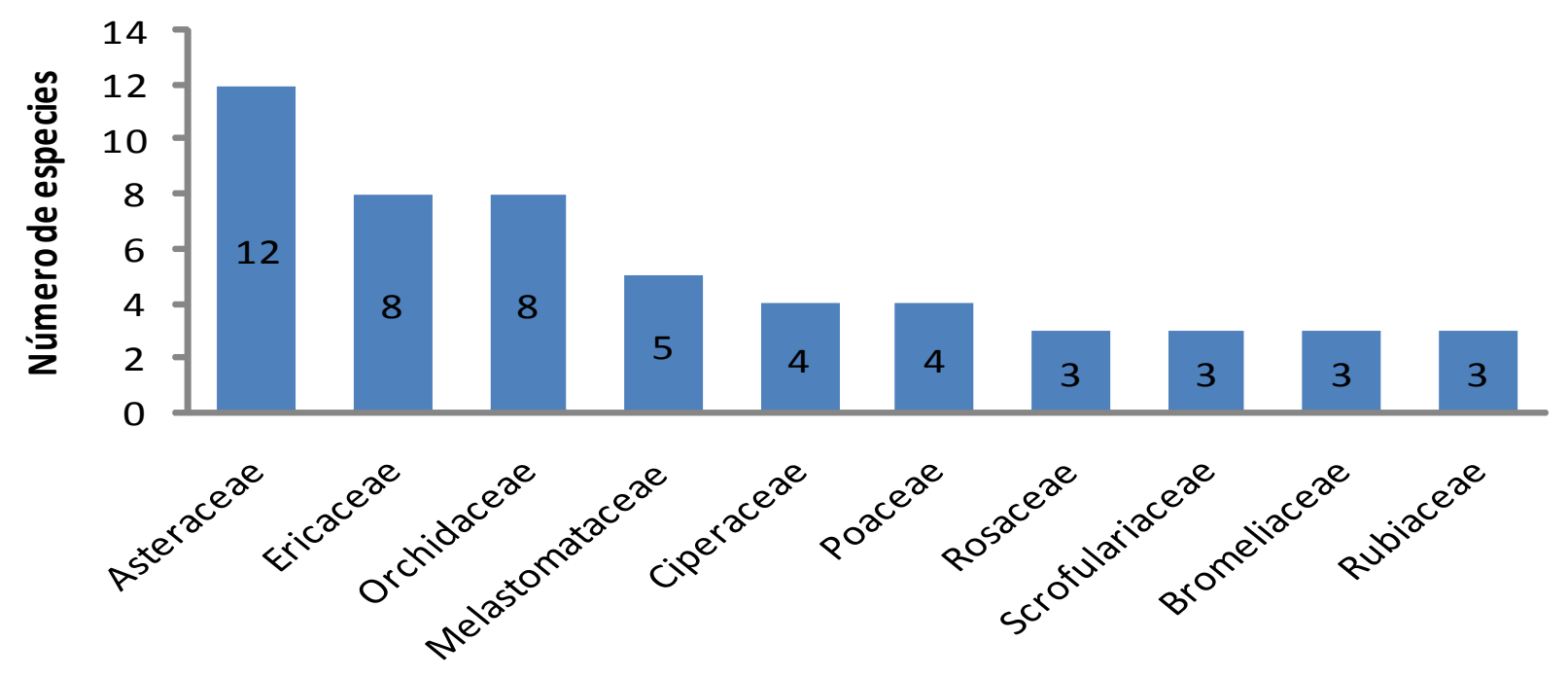

Familias Botánicas

Gráfica 1. Familias mejor representadas en el páramo del Duende.

mayor, los suelos son más drenados, lejos de las fuentes hídricas, en donde predominan bromelias terrestres que se convierten en retenedoras de agua; asociadas con estas poblaciones se encuentran hierbas de gran tamaño como pastos (Neurolepis sp.), helechos arbustivos Blechnum sp1 y algunos arbustos correspondientes a Melastomataceas (Miconia sp., Tibouchina grossa), Araliaceas (Sheflera sp.), compuestas, incluyendo frailejón en pequeñas proporciones; las alturas de los individuos varían entre $60 \mathrm{~cm}$ y $5 \mathrm{~m}$.

La distribución vertical de las especies presentes en el área correspondiente a la zona de vida páramo, permitió diferenciar varios tipos de hábitos: herbáceo, arbustivoy epifito, que dominan elárea de muestreo, predominando el herbáceo y arbustivo; los últimos conforman los bosques achaparrados propios de estos ecosistemas.

El estudio se realizó en diferentes sitios cercanos a las fuentes hídricas que integran el ecosistema paramuno; para un mejor entendimiento del esqueleto vegetal, la heterogeneidad del paisaje y la dinámica del ecosistema se tuvieron en cuenta tres áreas de muestreo: humedal Citará, ríos Cucurrupí y Copomá, en el nacimiento.

Flora en el humedal Citará. Este sitio

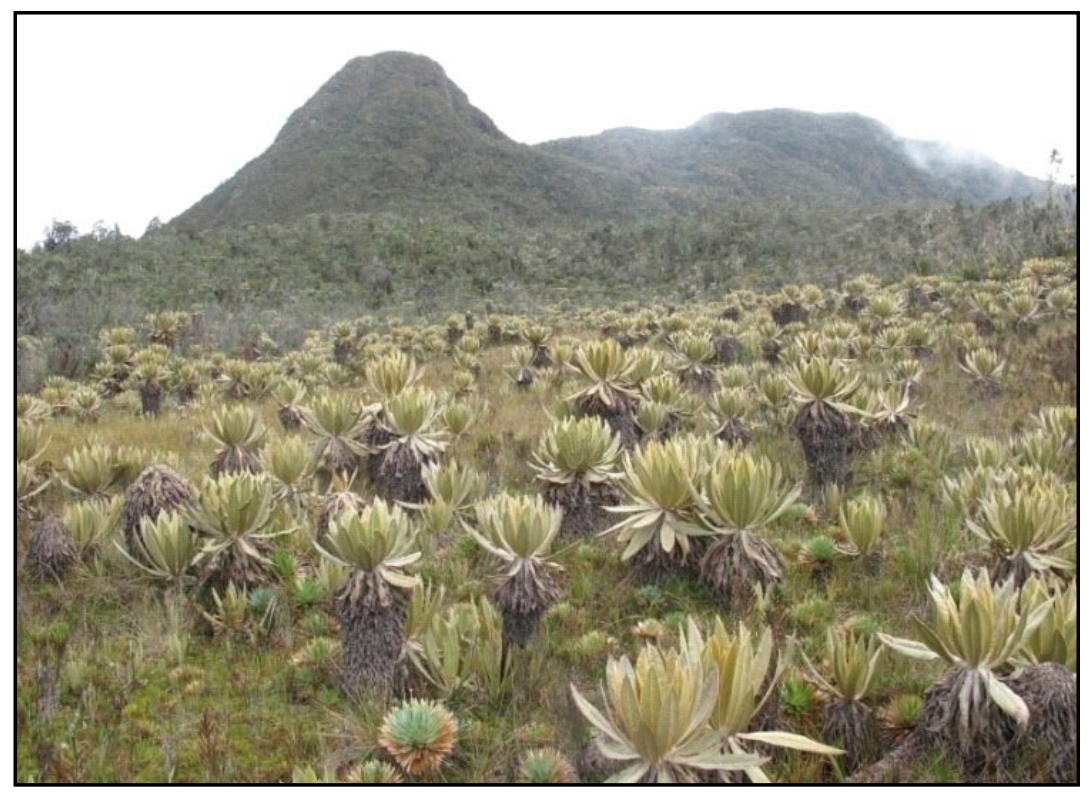

Figura 1. Vegetación abierta.

se caracterizó por presentar un espejo de agua reducido, debido quizá a la época de poca lluvia; los suelos circundantes se caracterizan por ser fangosos con una gruesa capa de materia orgánica (Figura $3 \mathrm{~A}$ ), lo que se debe a la humedad del área que por estar bordeada de pequeñas pendientes producen arrastre de biomasa descompuesta producida por la vegetación circundante; además se observaron afloramientos rocosos a lo largo de las pendientes.

Florísticamente esta área se encuentra representada sobre todo por poblaciones vegetales de Espeletia frontinoensis (frailejón), Loricaria complanata, hierbas con hojas arrosetadas de Paepalanthus colombianun, Valeriana bracteata, hierbas de porte erecto Castilleja fisifolia, Viola sp., Sisyrinchum 


\section{Bioetnia Volumen 7 № 2 (julio-diciembre), 2010}

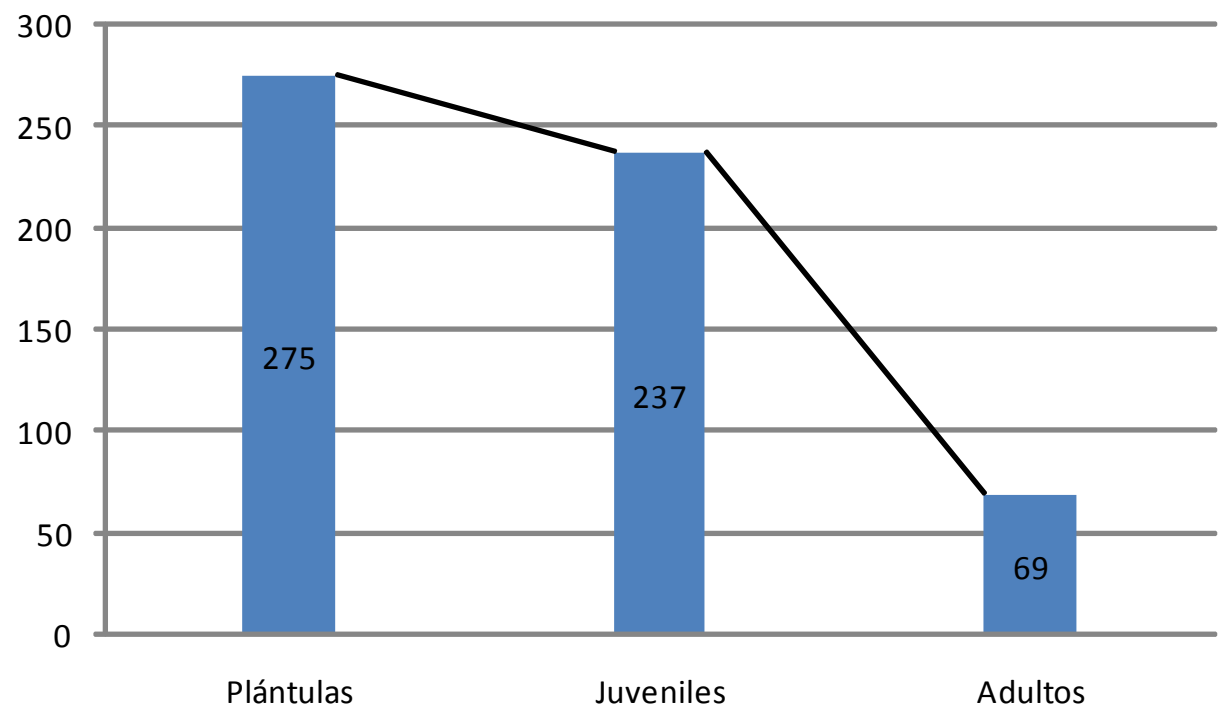

\section{Gráfica 2. Individuos de Espeletia frontinoensisen sus diferentes estados sucecionales en el páramo del Duende.}

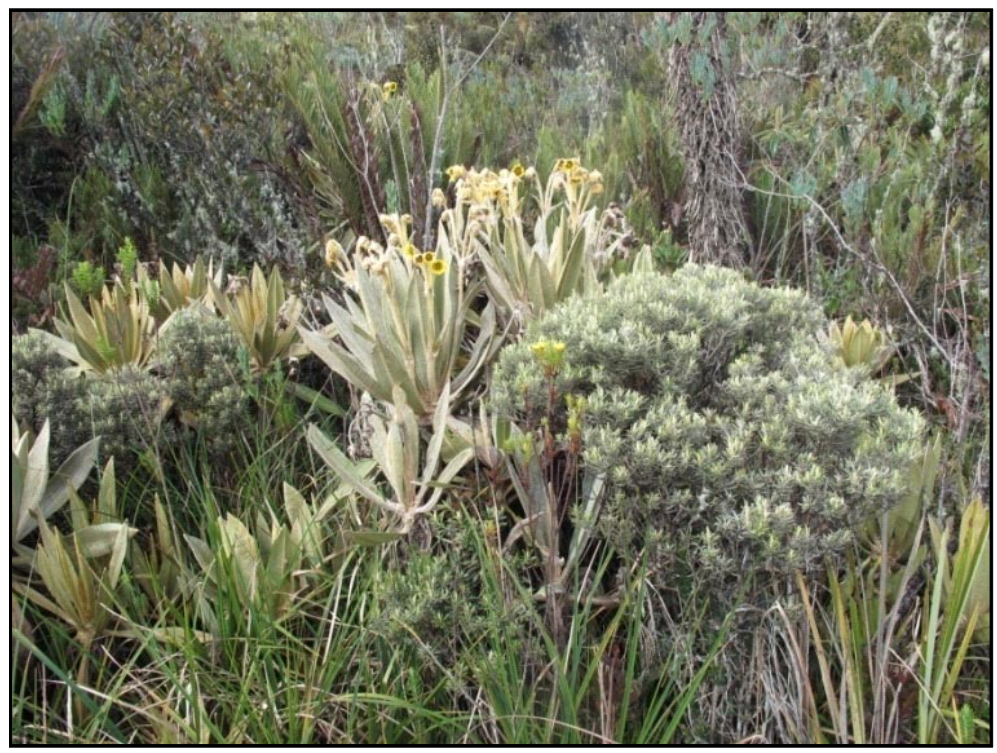

Figura 2. Vegetación arbustiva.

sp., Oritrophium cracifolium, acompañadas de hierbas de reducido tamaño que forman cojines como Xyris sp., Myrteola sp., Disterigma empetrifolium y asociaciones de pastizales donde se destacan representantes de las familias Poaceae y Cyperaceae acompañadas de musgos y helechos (Figura 3).

En los alrededores del humedal se observó un gran predominio de helechos correspondientes a Huperzia rufescens y Jamesonia sp., y algunos individuos de Epidendrun frutex y Neurolepis sp. En áreas cercanas a este humedal se observaron abundantes huellas de mamíferos posiblemente oso de anteojos, puma y tigrillo, acompañadas de excremento, lo que tal vez se debe a que estos sitios son tránsito de estas especies, que convergen desde la región Pacífica hasta la cordillera Central, convirtiendo a este ecosistema en un posible corredor.

Flora en el río Cucurrupí. El área se caracteriza por presentar suelos fangosos, poco drenados con abundante materia orgánica; florísticamente se encuentra representada por pastos correspondientes a las familias Cyperaceae (Rhynchospora) y Poaceae (Calamagrostis sp., Cortadeira sp., Fetusca sp.) entremezclados con pequeñas poblaciones de Lycopodyum clavatum, pequeños arbustos correspondientes sobre todo a las familias Asteraceae y Ericaceae; con menor frecuencia se observaron hierbas de porte erecto como Halenia drasyantha, Odontoglossum sp. y grandes poblaciones de E. frontinoensis (Figura $4 \mathrm{~A}$ ).

A unos metros del nacimiento del río, aunque la vegetación es muy similar, se observó en sus márgenes la presencia de arbolitos achaparrados de Sheflera sp. y Oreopanax sp., representantes de la familia Araliaceae, arbustos de Miconia sp., Tibouchina grossa y representantes de la familia Ericaceae, y en el estrato herbáceo se observó Peperomia rotundata, que se constituyen en alimento para la avifauna residente en este ecosistema (Figura 4 B).

La vegetación circundante de esta unidad muestral aporta materia orgánica producida por la hojarasca que se descompone y se constituye en hábitat, refugio y un buen recurso alimenticio para la entomofauna residente en el ecosistema. 

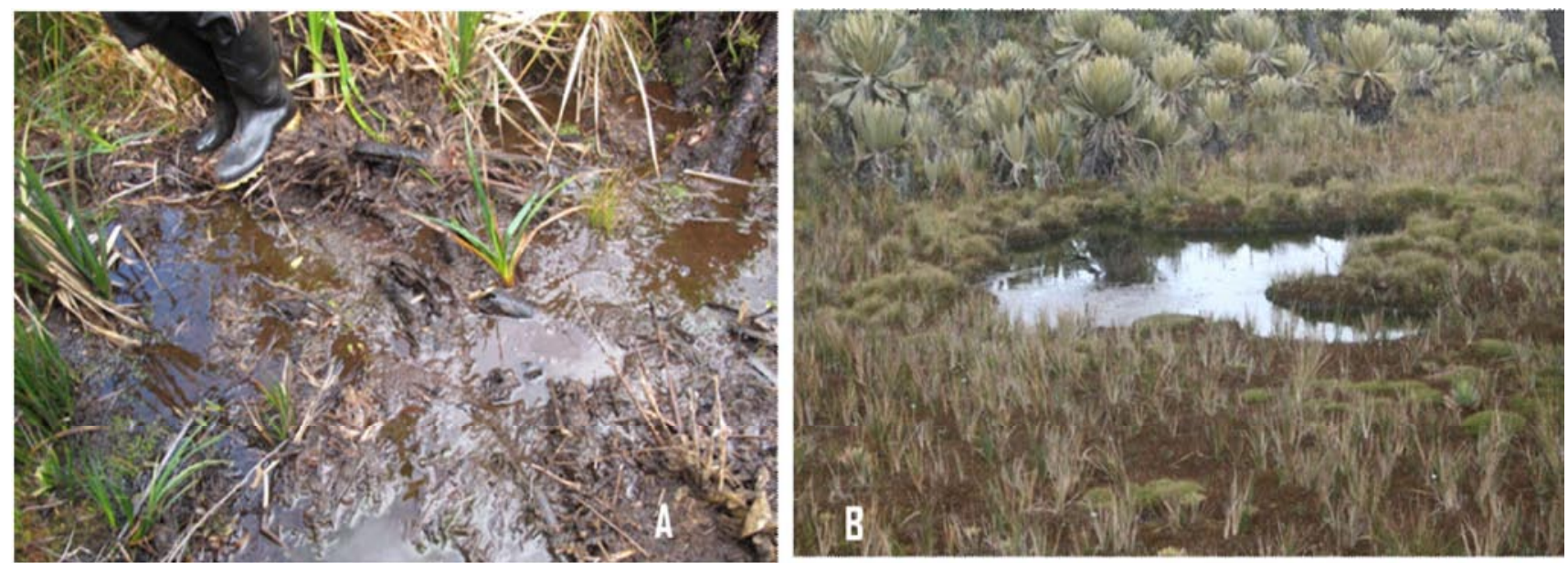

Figura 3. A. Área de pantano. B. Humedal Citará.

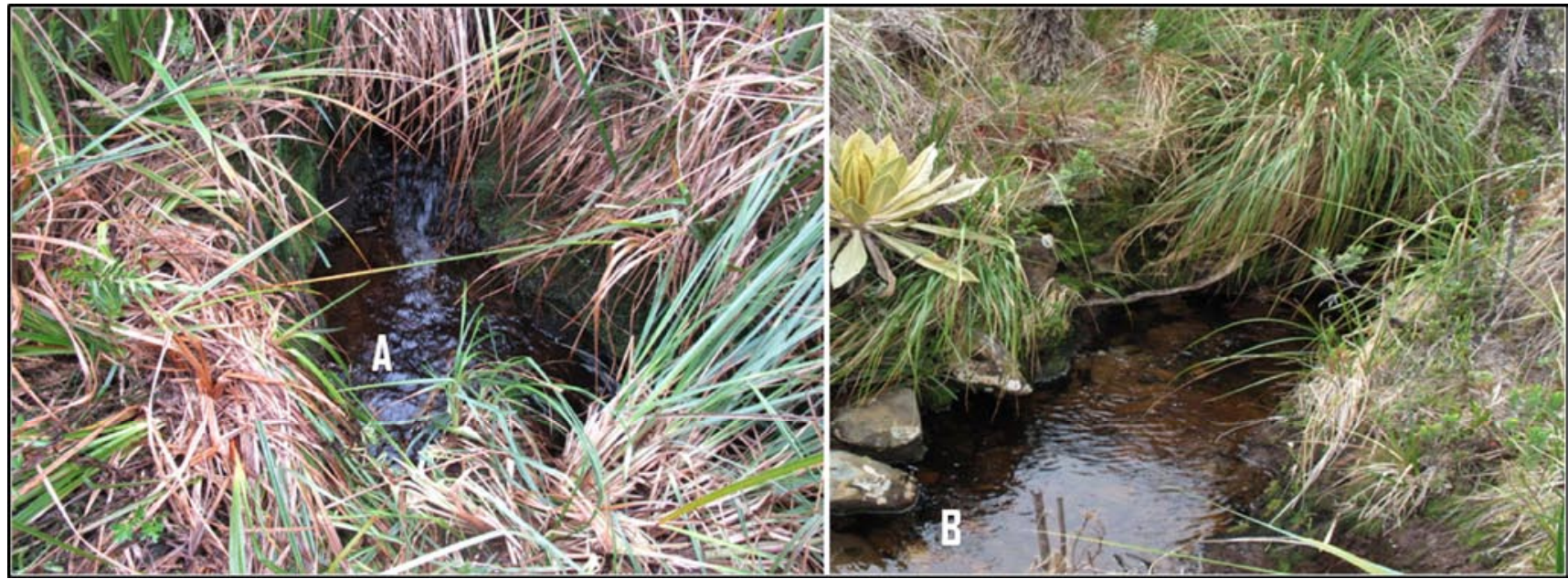

Figura 4. A. Nacimiento del río Cucurrupí. B. Aguas abajo del río Cucurrupí.

Flora en el río Copomá. La zona se caracteriza por presentar suelos fangosos, la vegetación circundante está compuesta por pastos correspondientes a las familias Cyperaceae (Rhynchospora) y Poaceae (Calamagrostis sp., Cortadeira sp., Fetusca sp.) entremezclados con pequeños arbustos pertenecientes sobre todo a las familias Asteraceae y Ericaceae; con menor frecuencia se observaron hierbas de porte erecto como Calceolaria sp., Gunnera scabra y hierbas de menor tamaño Lachemilla ssp. Se observó un estrato representado por musgos y hepáticas; en el estrato arbustivo fue muy frecuente la presencia de epifitas como orquídeas (Lephantes ssp., Pleurotalis pilifera, Palchyphyllum pastii, entre otras), bromelias, musgos, hepáticas y helechos; entre las especies y familias más representativas se encuentran Tibouchina grossa, Miconia ssp., Vallea stipularis así como representantes de las familias Ericaceae y Asteraceae, acompañadas de helechos arborescentes (Cyathea sp.) y frailejones de gran tamaño (20 m y $6 \mathrm{~m}$ ) que sobrepasan el nivel de dosel del esqueleto vegetal, situación que ratifica el buen estado de conservación y la antigüedad de este ecosistema, porque las dos especies se caracterizan por presentar un crecimiento vegetativo muy lento.

Esta área presentó un alto grado de epifitismo comparado con las áreas anteriores, lo posiblemente se deba a que el tramo muestreado no corresponde al nacimiento propiamente dicho y el arrastre de materia orgánica por parte de éste facilita el establecimiento de grandes poblaciones de especies arbustivas en las márgenes de este río.

En los alrededores de esta unidad muestral se observan un pequeño humedal y una quebrada, en los que dominan poblaciones de hierbas arrosetadas Plantago rigida, en un estrato arbustivo donde predomina E. frontinoensis. Asociados con estos cuerpos de agua se observaron con frecuencia poblaciones de Epidendrum fimbriatum, Laestadia sp., pe- 

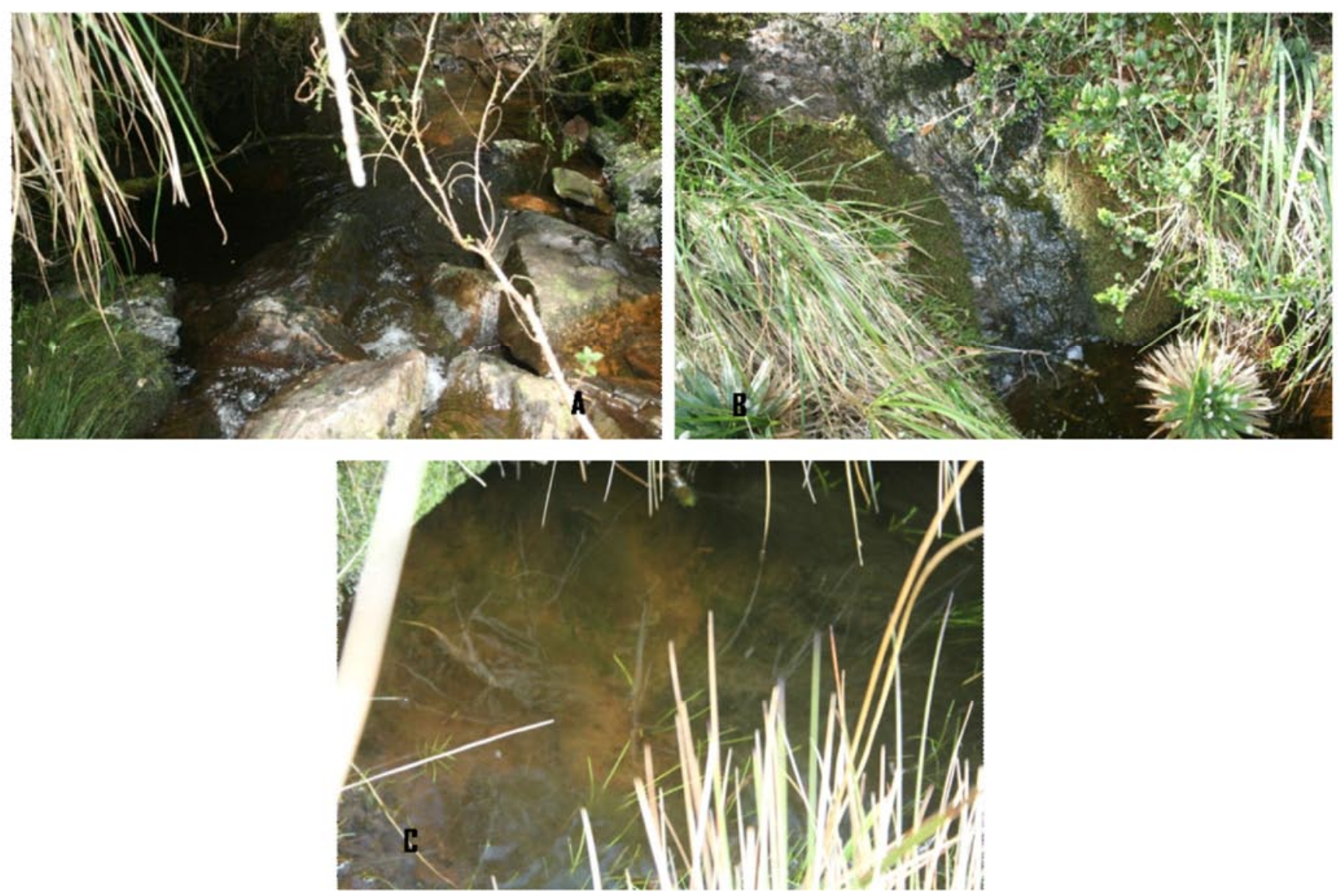

Figura 5. Zona de muestreo nacimiento del río Copomá.

queñas hierbas formando cojines como Nertera sp., disterigma empetrifolium, pastos de las familias Poáceas y Ciperáceas, asociadas con musgos y hepáticas (Figura 5).

Si se compara la representatividad de especies, género y familia para este estudio con los reportados para el mismo ecosistema jurisdicción del Valle del Cauca y ecosistemas similares, (Rangel 2000, CVC-FEDENA 2006, Rangel-Ch. y Sánchez 2005, Frontino, Murillo et al. 2008, Sanín et al. 2008, Gómezy Vargas 1999), el páramo del Duende jurisdicción del departamento del Chocó fue menos representativo en relación con los estudios mencionados, lo que se debe a un mayor nivel de estudio; sin embargo, la riqueza florística que se encontró en este estudio revela patrones por lo general similares a los encontrados en los estudios ya mencionados.

Al analizar la diversidad de familias, las Asteráceas, Poáceas, Ericáceas, Orquidáceas y Melastomatáceas se destacan como las más diversas no sólo en el Duende, sino en la mayoría de estudios de páramos del país.

Los recursos florísticos en estado de floración y fructificación presentes en la zona de páramo demuestran la oferta esencial para la existencia marcada de importantes grupos fáunicos (aves, insectos y mamíferos), lo que se pudo corro- borar, mediante la observación directa de aves e insectos. La oferta de estos recursos permite asumir la presencia de pequeños herbívoros, presas potenciales de los pumas, lo que evidencia la fortaleza de la estructura trófica del lugar. El viento dispersa gran parte de la vegetación presente en este ecosistema, lo que se evidenció por la dominancia de Asteráceas, Poáceas, Ciperáceas, Orchidáceas, entre otras; la presencia representativa de Ericáceas, Melastomatáceas, Araliáceas, con abundantes frutos (bayas) de reducido tamaño y de variados colores revelan otro importante medio de dispersión como es el realizado por la avifauna residente en el ecosistema.

El momento fenológico (floración) muestra la oferta de recurso para importantes y diversos grupos como los insectos y las aves y asimismo, la interacción entre algunas familias florísticas con esta fauna asociada, las que con corolas tubulares de taxones como Ericaceae, Desfontainiaceae, Scrophulariaceae, Alstromeriaceae, Gesneriaceae manifiestan el importante grado de relación con la fauna nectívora en especial con los colibríes.

La vegetación presente en áreas con mayor humedad cercanas a las fuentes hídricas (humedales, quebradas y ríos) 
muestra patrones diferentes por completo a la vegetación presente en las áreas alejadas de las fuentes ya mencionadas. Cerca a estas fuentes de agua las especie más abundantes son E. frontinoensis, que forma grandes parches, los pastizales conformados por Camalagrostis sp., Fetusca sp., Rinchospora sp. mientras que en pendientes y colinas alejadas de estas fuentes predominan especies Guzmania sp., Blechnum sp., Epidendrum frutex, Neurolepis sp.; lo anterior concuerda con lo manifestado por CVC-FEDENA(2006).

\section{CONCLUSIONES}

La riqueza florística que se encontró en el páramo del Duende en jurisdicción del departamento del Chocó, mostró patrones similares a los encontrados en el mismo ecosistema en jurisdicción del Valle del Cauca.

La falta de identificación del material colectado en campo no ha permitido hasta la fecha tener un dato de las especies presentes en el ecosistema que se encuentran con algún grado de amenaza o listadas en los libros rojos de Colombia o especies endémicas.

El páramo del Duende es un ecosistema que se encuentra en muy buen estado de conservación, lo que se puede deber a factores como su difícil acceso, la cultura de los pueblos asentados en las partes bajas de los ríos que allí nacen, factores climáticos como alta radiación solar y bajas temperaturas nocturnas, sumado a la topografía del terreno que no permitirían el establecimiento de poblaciones humanas en el ecosistema paramuno.

\section{LITERATURACITADA}

CVC-FEDENA. 2006. Informe final de la formulación del plan de manejo del PNR Páramo del Duende y su zona amortiguadora. Convenio $\mathrm{N}^{\circ} 178 \mathrm{de}$ 2005. Cali CVC-FEDENA; 216 p.

Rangel-CH, O. 2000. Visión integradora sobre la región del páramo. En: Rangel, J. O. Colombia Diversidad Biótica III. La Región de Vida Paramuna. Bogotá, DC: Universidad Nacional de Colombia-Instituto de Ciencias Naturales Alexander von Humboldt; p. 1-23.

Hotstede, R., P. Segarra, P. Mena V. (eds.) 2003. Los páramos del mundo. Proyecto Atlas Mundial de los Páramos. Quito: Global Peatland Iniciative/ NC-UICN/Ecociencia; 299 p.

Rangel, J. O. 2007. La región paramuna en Colombia y en la serranía del Perijá. En: Rangel, J. O. Colombia Diversidad Biótica V. La Alta Montaña de la Serranía del Perijá. Bogotá, DC: Universidad Nacional de ColombiaInstituto de Ciencias Naturales Corporación Autónoma Regional del CésarCORPOCESAR; p. 1-18.

Sklenár P., J. L. Luteyn, Ulloa, U. C., Jorgensen P. M., Dillon, M. O. 2005. Flora génerica de los páramos. Guía ilustrada de plantas vasculares. Volumen 92. New York: Memories of the New York Botanical Gardens; 520 pp.

Vargas W. G., Gómez H. N. 2005. Páramo del Duende (cordillera Occidental): Datos sobre flora y fauna. En: Van Der Hammen, T. J., Rangel, O., Cleaf, A. M. Estudios de ecosistemas tropoandinos-la cordillera Occidental colombiana. Transepto Tatamá. Proyecto Ecoandes. Berlin: Stuttgart: J. Cramer Inder Gerb. Borntraeger Ver Lags Buchhandlung; p. 972. 